\title{
Multimodality Functional Imaging in Radiation Therapy Planning: Relationships between Dynamic Contrast-Enhanced MRI, Diffusion-Weighted MRI, and 18F-FDG PET
}

\author{
Moisés Mera Iglesias, ${ }^{1}$ David Aramburu Núñez, ${ }^{1}$ José Luis del Olmo Claudio, ${ }^{1}$ \\ Antonio López Medina, ${ }^{1}$ Iago Landesa-Vázquez, ${ }^{2}$ Francisco Salvador Gómez, \\ Brandon Driscoll, ${ }^{3}$ Catherine Coolens, ${ }^{3}$ José L. Alba Castro, ${ }^{2}$ and Victor Muñoz ${ }^{4}$ \\ ${ }^{1}$ Medical Physics Department and Radiological Protection, Galaria-Hospital do Meixoeiro-Complexo Hospitalario \\ Universitario de Vigo, 36200 Vigo, Spain \\ ${ }^{2}$ Signal Theory and Communications Department, University of Vigo, 36310 Vigo, Spain \\ ${ }^{3}$ Radiation Medicine Program, Princess Margaret Cancer Centre and University Health Network, Toronto, ON, Canada M5T 2 M9 \\ ${ }^{4}$ Radiation Oncology Department, Galaria-Hospital do Meixoeiro-Complexo Hospitalario Universitario de Vigo, 36200 Vigo, Spain
}

Correspondence should be addressed to Antonio López Medina; antonio.lopez.medina@sergas.es

Received 4 July 2014; Revised 15 September 2014; Accepted 10 October 2014

Academic Editor: Alexandru Dasu

Copyright (C) 2015 Moisés Mera Iglesias et al. This is an open access article distributed under the Creative Commons Attribution License, which permits unrestricted use, distribution, and reproduction in any medium, provided the original work is properly cited.

Objectives. Biologically guided radiotherapy needs an understanding of how different functional imaging techniques interact and link together. We analyse three functional imaging techniques that can be useful tools for achieving this objective. Materials and Methods. The three different imaging modalities from one selected patient are ADC maps, DCE-MRI, and 18F-FDG PET/CT, because they are widely used and give a great amount of complementary information. We show the relationship between these three datasets and evaluate them as markers for tumour response or hypoxia marker. Thus, vascularization measured using DCEMRI parameters can determine tumour hypoxia, and ADC maps can be used for evaluating tumour response. Results. ADC and DCE-MRI include information from 18F-FDG, as glucose metabolism is associated with hypoxia and tumour cell density, although 18F-FDG includes more information about the malignancy of the tumour. The main disadvantage of ADC maps is the distortion, and we used only low distorted regions, and extracellular volume calculated from DCE-MRI can be considered equivalent to ADC in well-vascularized areas. Conclusion. A dataset for achieving the biologically guided radiotherapy must include a tumour density study and a hypoxia marker. This information can be achieved using only MRI data or only PET/CT studies or mixing both datasets.

\section{Introduction}

Radiotherapy is in a process of transformation from imageguided radiotherapy to biologically guided radiotherapy [1] To this effect, in the last few years some commercial treatment units have been developed that include an MRI unit combined with a linac in a single device [2-5], and PET/CT (positron emission tomography/computed tomography) has proven useful for tumour staging and target delineation, especially in head and neck tumours and lung tumour [68]. The main change in clinical practice will arrive when the prescription of a treatment moves from dose prescribed to target volumes and to prescription of results of a function, as tumour control probability, which considers all the radiobiological phenomena and adapts the treatment to early tumour response and uses different functional images as inputs. Although not widely available yet, several tools, methods, and models have been developed for achieving these objectives in a retrospective manner:

(1) quantitative methods in diffusion-weighted imaging(DW-) MRI providing ADC (apparent diffusion coefficient) maps that allow determining early tumour response [9-13], 
(2) in vivo measurement of hypoxia [14, 15], using either MRI datasets [16-22] or PET/CT [23-28],

(3) inverse-planning optimisation algorithm that includes biological criteria $[29,30]$ and/or functional imaging information [31,32] or even radiobiological models adapted to functional imaging information [33].

In this paper a case study is presented using datasets from 18F-FDG (fludeoxyglucose labelled with 18F) PET/CT, DWMRI/ADC maps, and dynamic contrast-enhanced- (DCE-) MRI for characterizing tumour behaviour and for using the multimodality parameters as predictive values of tumour response from a patient included in the ARTFIBio project [34-36].

18F-FDG PET images the glucose consumption of each region. Tumour cells use glycolysis rather than lipolysis as the metabolic process to produce ATP and they use more glucose than normal cells. Glycolysis is a rather inefficient process and therefore large amounts of glucose are needed for cell survival and tumour growth. The PET enhancement (standard uptake value or SUV) in tumours is due to three different mechanisms: (i) cancer cells produce more ATP outside the mitochondria, even in well-oxygenated conditions (Warburg effect [37]); (ii) cancer cells proliferate more than normal tissue cells [38], and then they need more glucose; and, finally, (iii) cancer cells can survive in lower oxygenated regions better than normal tissue cells $[39,40]$ but consume more glucose because they need to produce ATP by glycolysis in absence of oxygen (Pasteur effect).

DW-MRI measures the diffusion of protons in a medium. Its principle is based on the attenuation of the signal according to Stejskal and Tanner's model [41]. Tumour cells are abnormal in size and shape compared to normal cells, and they are more tightly packed and have higher cellularity than the tissue from which they originate. The extracellular volume is smaller in tumour regions, and therefore the freedom of movements of protons in tumour regions is restricted [42, 43]. The logarithm of the signal attenuation is a function of the applied gradient, the gap between pulses of gradient, and the pulse duration. By varying these parameters during acquisition, the ADC can be calculated for each voxel.

DCE-MRI has been proposed by several authors for treatment monitoring [44-46] and for measurement of oxygenation distribution [19-22]. The main problem is the complexity of the data analysis and the correspondence between measurement and biological parameters. Another disadvantage is the necessity of a contrast agent.

In order to characterize the tumour and to implement new predictive models based on functional imaging data, we must ensure we can extract as much information as possible from the available data. Some of the main parameters to characterize tumour behaviour, along with radiotherapy treatment, must be initial tumour density, hypoxia, malignancy/ proliferation, dose to each voxel, and timing of the dose. In this work, attention is focused on showing the relationship between ADC maps, DCE-MRI parameters, dose, and 18F-FDG PET/CT SUV (standard uptake value). Many other types of images can show the main parameters we are interested in modelling (18F-fluorothymidine for proliferation
[47], Zr-89-cetuximab for response to chemotherapy [31], and dynamic FDG [28] and fluoromisonidazole (FMISO) [26] for hypoxia), but it is hypothesized that the proposed combination of techniques can give us enough information about the tumour environment to assess the treatment response, but not the tumour microenvironment (data are averaged into the voxel size): the $k_{\text {trans }}$ parameter in DCEMRI is related to vascularization and then to hypoxia [18] and $v_{e}$ is related to extracellular volume and in heterogeneously vascularized areas to tumour density [18]; SUV is related to tumour metabolism and then is related to malignancy (enhancement of the Warburg effect to the Pasteur effect), hypoxia (Pasteur effect), and tumour density and proliferation. Finally, ADC maps are related to water mobility and then to tumour density [12]. We will explore the relationships between ADC, DCE-MRI parameters, and SUV values and will evaluate their influence on tumour response in a case study where we have in the same slice a necrotic volume, a hypoxic area, and a heterogeneously vascularized tumour volume.

\section{Material and Methods}

2.1. Patients. This study is conducted in accordance with the Declaration of Helsinki [48] and the study protocol was approved by the local ethics committee; informed consent was obtained from all patients.

The aim of ARTFIBio project (http://artfibio.cesga.es/Artfibio/application/) is to create a network for sharing information and for developing predictive individualized models of the tumour response to radiotherapy in patients with head and neck cancer based on in vivo functional data. For this purpose, several studies of MRI and PET/CT were performed. Patients within the ARTFIBio project [34-36] had oropharyngeal cancer (squamous cancer cell) of stages T3 and T4. All of them are treated with IMRT (intensity-modulated radiation therapy) and the prescribed dose was between 66 Gy and 70 Gy to the local PTV. The imaging protocol (Figure 1) is as follows:

(i) pretreatment: MRI study (DCE-MRI + ADC) and PET/CT study (18F-FDG),

(ii) first control (10-30 Gy): MRI study (DCE-MRI + $\mathrm{ADC}$ ),

(iii) second control (30 Gy-60 Gy): MRI study (DCE$\mathrm{MRI}+\mathrm{ADC})$

(iv) three months after the treatment: PET/CT and MRI study (DCE-MRI + ADC).

For all imaging studies the patient is positioned using the RT immobilisation devices. The geometrical distortion on MRI images and registration process (rigid registration and deformable registration) were checked with an MRI phantom. Regardless, only central slices showing low distortion were analysed. For each patient and each set of images the ADC values, contrast exchange coefficients $\left(K_{\text {trans }}\right)$, SUV, dose, and Hounsfield units (HU) per voxel were recorded of each volume. 
TABLE 1: Main parameters of MRI acquisition protocols.

\begin{tabular}{|c|c|c|c|c|c|c|c|}
\hline Technique & $\begin{array}{c}\text { TR/TE } \\
(\mathrm{ms})\end{array}$ & $\begin{array}{l}\text { Field of view } \\
\qquad\left(\mathrm{cm}^{2}\right)\end{array}$ & Matrix size & $\begin{array}{c}\text { Slice } \\
\text { thickness } \\
(\mathrm{mm})\end{array}$ & Gap & $\begin{array}{l}\text { Sense } \\
\text { factor }\end{array}$ & $\begin{array}{c}\text { Contrast } \\
\text { agent }\end{array}$ \\
\hline T1-Turbo Spin Echo & $425 / 4.8$ & $23 \times 23$ & $272 \times 272$ & 6 & 1 & 1.6 & - \\
\hline T2-Turbo Spin Echo & $6171 / 90$ & $23 \times 23$ & $320 \times 312$ & 6 & 1 & 1.6 & - \\
\hline $\mathrm{ADC} b=0,600 \mathrm{~s} / \mathrm{mm}^{2}$ & $5270 / 77$ & $25 \times 25$ & $120 \times 97$ & 6 & 1 & & - \\
\hline $\mathrm{ADC} b=0,1000 \mathrm{~s} / \mathrm{mm}^{2}$ & $5926 / 85$ & $25 \times 25$ & $120 \times 97$ & 6 & 1 & & - \\
\hline $\begin{array}{l}\text { DCE-MRI-Dynamic T1 High Resolution Isotropic } \\
\text { Volume Excitation (THRIVE) }-7 \text { series every } 33 \mathrm{~s}\end{array}$ & $4.1 / 1.97$ & $24 \times 24$ & $120 \times 120$ & 6 & & 1.5 & Gd \\
\hline
\end{tabular}
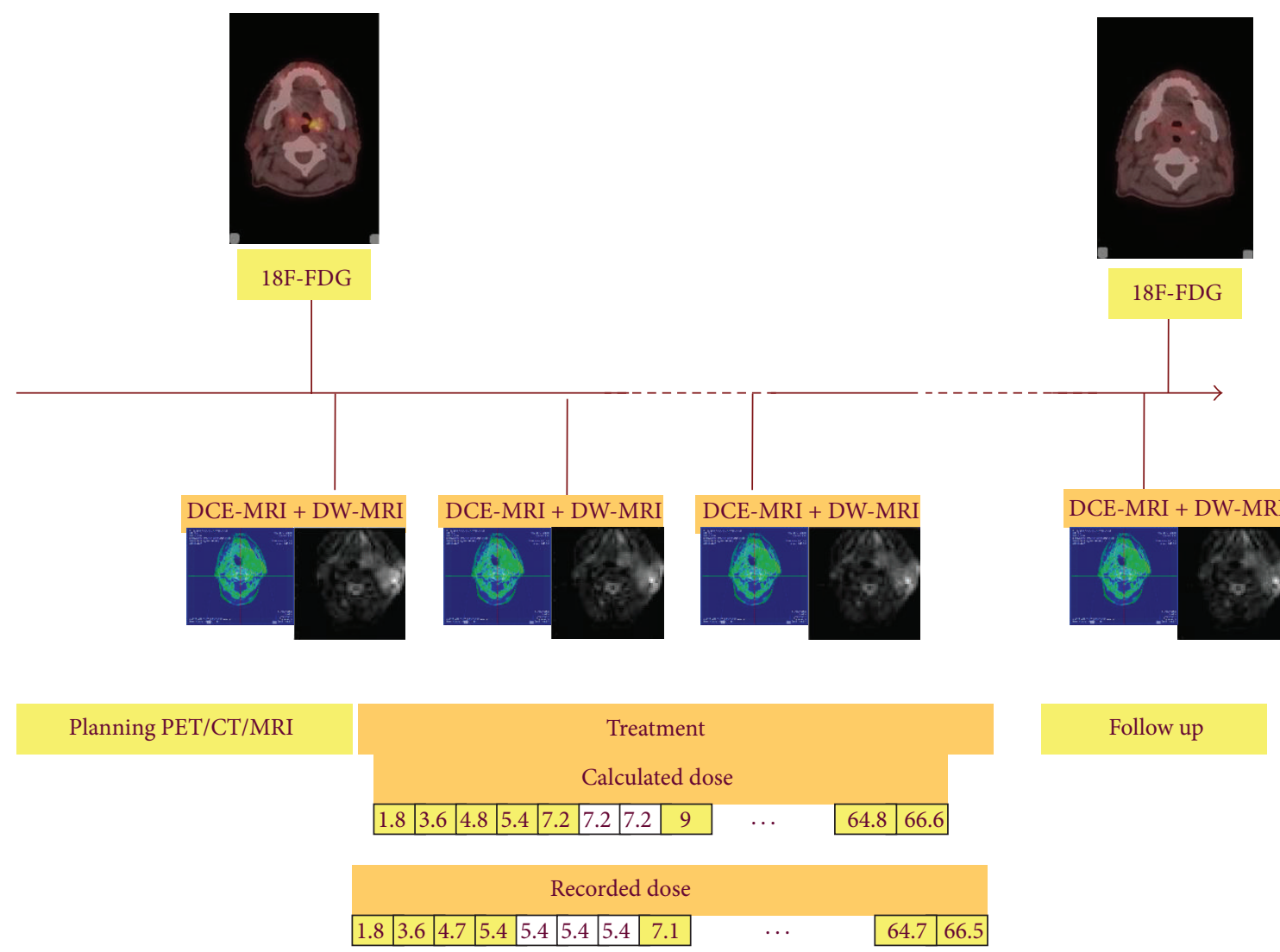

FIGURE 1: Scheme of the image acquisition process along the radiotherapy course.

In this paper a case study is highlighted from one patient who has three clearly differentiated volumes in a single slice: a heterogeneously vascularized tumour and a hypoxic region surrounding a necrotic area. This case is very useful to visualize and investigate the different behaviours of the tumour volumes in glucose metabolism and in treatment response.

2.2. Acquisition and Analysis of MR Images. All MRI examinations were performed on a 1.5-T scanner (Achieva; Philips Healthcare) with the patients in supine position. Routine T2-weighted, T1, DW-MRI, and DCE-MRI were obtained using the parameters showed in Table 1. Flex-L coil (Philips Sense Flex Medium) was placed over the neck. After image acquisition, pixel-to-pixel ADC map was reconstructed using the standard software on the imaging console (Achieva; Philips Healthcare). According to Stejskal and Tanner's model [41] and considering the monoexponential approximation, the ADC value can be calculated using the following:

$$
\mathrm{ADC}=\frac{\ln \left(S_{0} / S_{1}\right)}{\left(b_{1}-b_{0}\right)},
$$

where $S_{1}$ and $S_{0}$ are signal values of the images at $b$ values, $b_{1}$ and $b_{0}$, respectively, and ADC is the apparent diffusion coefficient obtained using $b_{1}=600$ and $b_{0}=0$.

A nonlinear model [49] was utilized to convert signal to gadolinium concentration in DCE-MRI as per Tofts [50]. It considers two different compartments: the blood plasma (or intravascular space) and the extracellular extravascular space 
TABLE 2: Main parameters of the Tofts model.

\begin{tabular}{lc}
\hline Quantity & Definition \\
\hline$C_{a}(t)$ & Arterial concentration as a function of time \\
$C_{t}(t)$ & Tissue concentration as a function of time \\
$H_{c t}$ & Hematocrit volume \\
$K_{\text {trans }}$ & Transfer constant from the blood plasma into the EES \\
$K_{e p}$ & Transfer constant from the EES back to the blood plasma \\
$T$ & Onset time of arterial contrast uptake \\
$v_{b}$ & Whole blood volume per unit of tissue \\
$v_{e}$ & Total EES volume $\left(v_{e}=K_{\text {trans }} / K_{e p}\right)$ \\
\hline
\end{tabular}

(EES or interstitial space). The parameters utilized to generate the Tofts model are described in Table 2.

The relationship between all these parameters can be obtained by

$$
C_{t}(t)=\frac{K_{\text {trans }}}{1-H_{c t}}\left(C_{a}(t) \otimes e^{-K_{e p}(t-\tau)}\right)+V_{b} C_{a}(t) .
$$

A voxel-based perfusion analysis method was used based on the modified Tofts model [49]. A 3D voxel-wise perfusion analysis method $[51,52]$ was applied to the DCE-MRI data which generated perfusion parameters $k_{\text {trans }}, k_{e p}$, and $v_{b}$ from the modified Tofts model. This method also provided semiquantitative metrics such as area under the curve (AUC) and time to max enhancement.

Variable flip angle (VFA) spoiled gradient recalled echo scans at three flip angles variations $\left(5^{\circ}, 10^{\circ}\right.$, and $\left.15^{\circ}\right)$ were utilized to calculate the voxel by voxel $\mathrm{Tl}_{0}$ of the GTV (gross tumour volume) of 3 different patients. The average $\mathrm{T}_{0}$ of these patients ( $800 \mathrm{~ms}$ ) was applied when calculating the concentration of the analyzed patient which unfortunately did not have VFA scans themselves.

The arterial input function (AIF) was chosen in the carotid artery near the base of skull.

\subsection{Acquisition of PET/CT Images. Whole-body PET/CT} scan was carried out from head to thigh, $60 \mathrm{~min}$ after intravenous administration of approximately $370 \mathrm{MBq}( \pm 10 \%)$ of $18 F-F D G$ on a PET/CT scanner (Discovery, GE Healthcare Bio-Sciences Corp.) with a $70 \mathrm{~cm}$ axial FOV, a $218 \times 218$ matrix. Study was acquired in $3 \mathrm{D}$ mode. The pixel spacing was $5.47 \mathrm{~mm}$ with a slice thickness of $3.27 \mathrm{~mm}$. The spatial resolution to $1 \mathrm{~cm}$ varies from $3.99 \mathrm{~mm}$ to $4.56 \mathrm{~mm}$. PET images were corrected for attenuation, scatter, decay, dead time, random coincidences, and slice sensitivity.

To calculate the SUV [53] for the selected patient and on a voxel by voxel basis, we took into account an injected activity of $345 \mathrm{MBq}$ with a weight of the patient of $49 \mathrm{~kg}$.

2.4. Noise Reduction and Registration. To reduce image noise a $3 \times 3$ nearest-neighbour smoothing filter was applied to the DCE-MRI, PET-CT, and ADC images. Deformable registration of the images, with the CT of treatment as reference, was performed using tailored in-house software specifically developed for the ARTFIBio project [36] and based on ITK

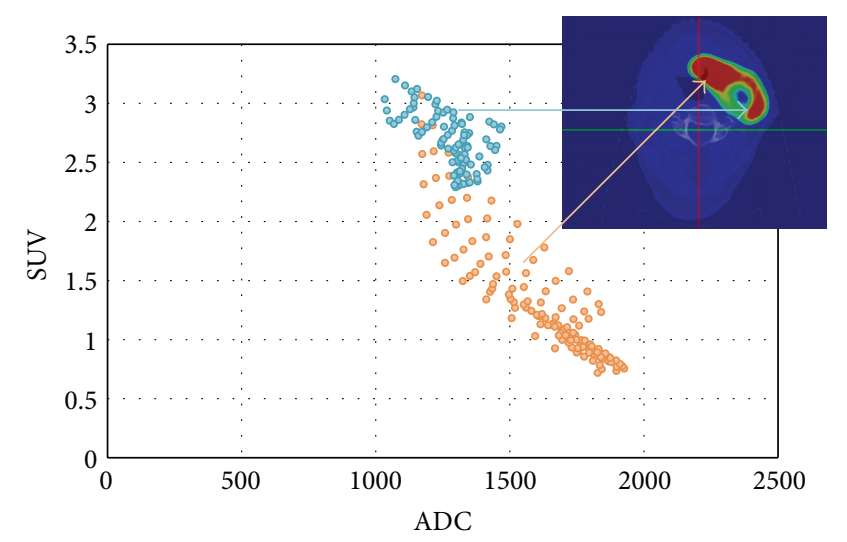

- Tumor away from necrosis area
- Near necrosis area

FIGURE 2: The relationship between SUV and ADC. In the hypoxic area (excluding necrotic area), high SUV values are obtained independently of the ADC value; this is explained by the addition of the Warburg effect and the Pasteur effect. In the heterogeneously vascularized area, SUV values decrease with ADC. This is likely a result of the fact that a reduction in ADC implies an increase in tumour cell density.

libraries [54]. Using the GTV contoured for radiation treatment, the numerical values of each voxel of the coregistered images were extracted. Bone and air voxels (as determined by CT) were dropped from the analysis profiles.

\section{Results and Discussion}

3.1. SUV versus ADC. The relationship between the different image datasets and functional parameters was investigated in order to achieve the best possible picture of the internal tumour dynamics. Using one representative patient a plot of SUV versus ADC for the CTV is displayed in Figure 2; the hypoxic area (low $\mathrm{ADC}$, low $K_{\text {trans }}$ ), surrounding necrotic volume (medium-high ADC, low $K_{\text {trans }}$ ), and heterogeneously vascularized tumour (low ADC, high $K_{\text {trans }}$ ) have each been considered separately (Figure 2).

3.2. ADC versus $v_{e}(D C E-M R I)$. Several parameters can be obtained from DCE-MRI, but only the relationships between $K_{\text {trans }}$ and extracellular volume $v_{e}$ have been investigated here.

In order to perform kinetic modeling of the tumour robust arterial input function (AIF) needs to be selected.

The AIF was chosen in the carotid artery near the base of skull for increased reproducibility since a larger variability was observed in the values of $\mathrm{Tl}_{0}$ in the carotid at the level of the neck (Figure 3).

$v_{e}$ should be most closely correlated to ADC information as the extracellular volume is related to the freedom of water molecules in the medium. Both sets of data were compared and they are represented in Figure 4.

For values of $v_{e}$ greater than 0.02 (values less than this value correspond to badly vascularized areas and low $K_{\text {trans }}$ in the studied data), a clear relationship between both datasets 


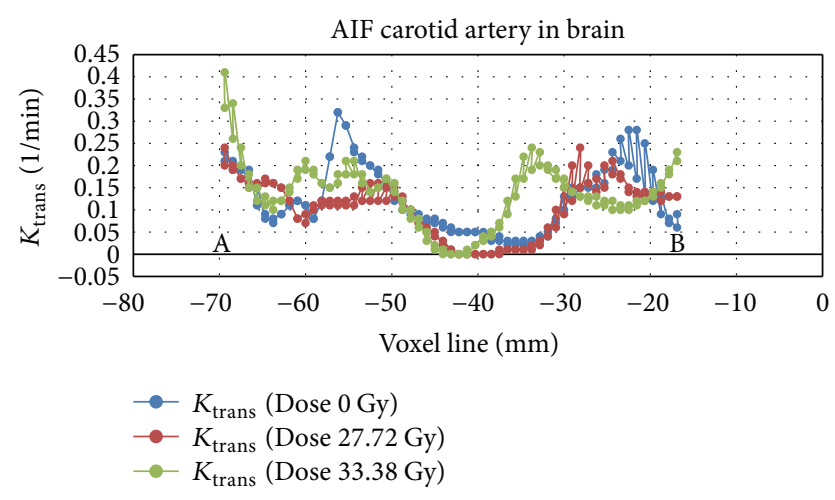

(a)

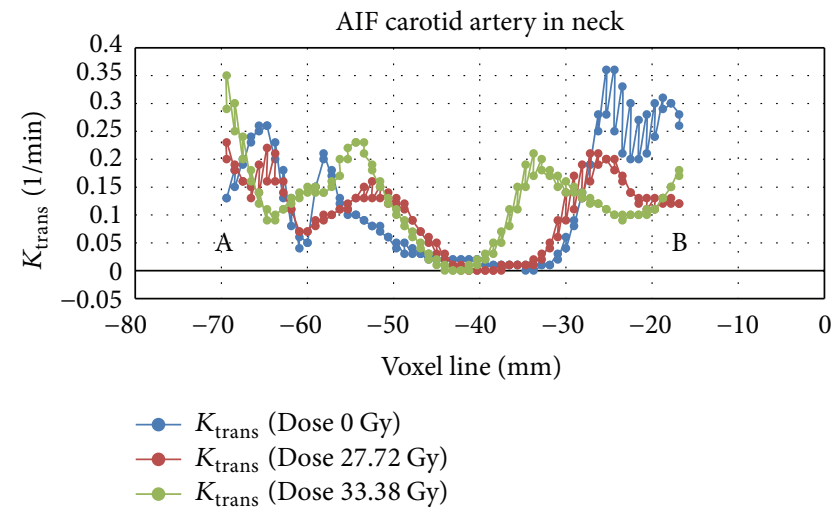

(b)

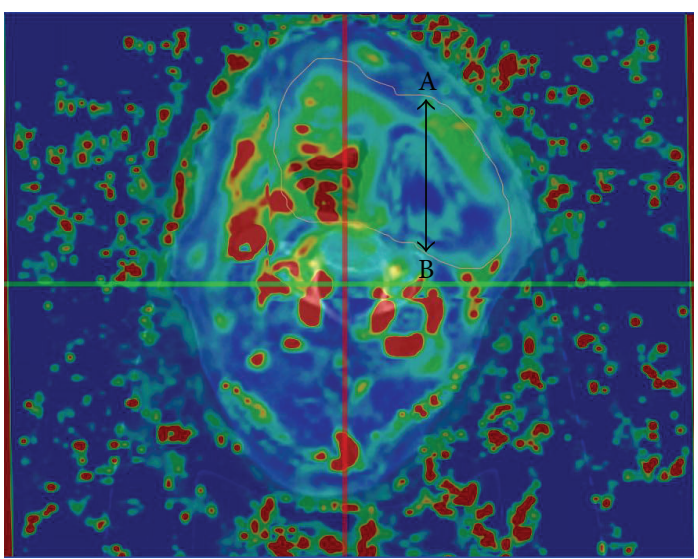

(c)

FIGURE 3: In this axial slice (bottom), $k_{\text {trans }}$ is represented along (top) a voxel line at different stages of treatment (pretreatment, at 27.72 Gy, and at $33.38 \mathrm{~Gy}$ ). We can see how $k_{\text {trans }}$ increases with dose and the central U-shaped valley corresponding to the badly vascularized area is becoming increasingly narrow. In the upper left figure, we consider AIF from data of the carotid artery near brain, and in the lower left figure, we consider AIF from data of the carotid artery in neck.

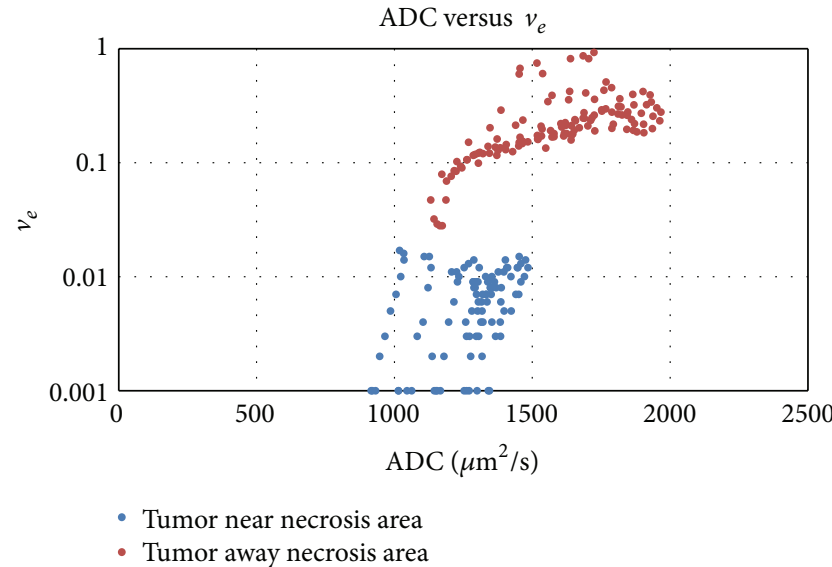

FIGURE 4: $v_{e}$ versus ADC $\left(\mu \mathrm{m}^{2} / \mathrm{s}\right)$ for the selected slice of the patient of Figure 3. In well-vascularized areas (red dots), a clear relationship can be found.

is found, indicating that a smaller extracellular volume corresponds to a higher tumour cell density in well-vascularized or heterogeneously vascularized areas but not hypoxic areas.
3.3. SUV, ADC versus $k_{\text {trans }}$ (DCE-MRI). Of all the analyzed parameters, $k_{\text {trans }}$ is the most related to vascularization. Vascularization must be related to oxygenation [55-59], as Figure 5 shows, because with increasing $K_{\text {trans }}$ values, that is, increasing perfusion, SUV values decrease because of the reduction of the Pasteur effect (green dots, Figure 5(c)).

On the other hand, no clear relationship has been found between ADC map and $k_{\text {trans }}$ values, although ADC values appear to be rather constant (blue dots, Figure 5(d)) because they are selected from a small homogeneous region. Additionally, tumour cells are able to survive in badly oxygenated areas and the tumour cell density is less variable in these areas.

3.4. ADC versus Dose Influenced by $K_{\text {trans }}$. We have generated the ADC values during the treatment for a heterogeneously vascularized tumour volume. In this case, the delivered dose to achieve an ADC value corresponding to normal tissue is much lower than for badly vascularized voxels. The influence of vascularization/oxygenation in the ADC response can be observed with the DCE-MRI studies, as shown in Figure 6.

3.5. Discussion. The results presented have some similarities to those obtained by Atuegwu et al. [12] and indicate that 


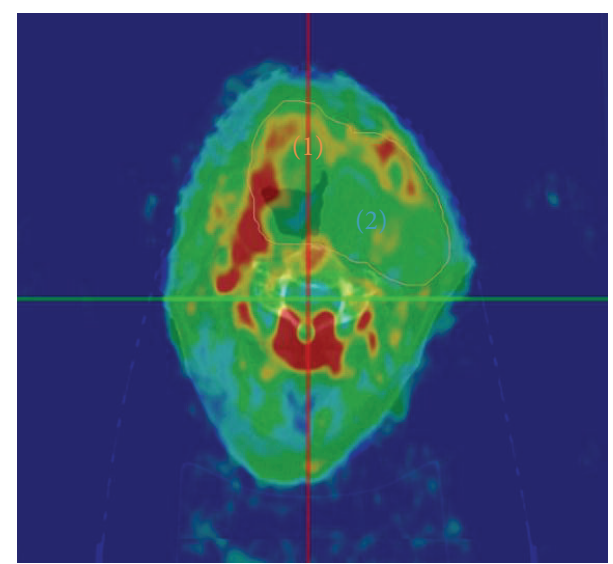

(a)

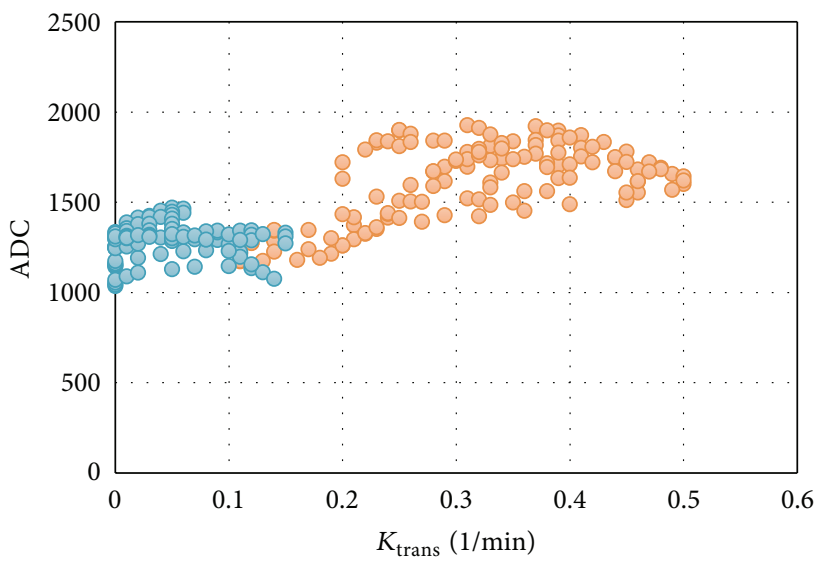

- Tumor away to necrosis area ${ }^{(1)}$

Near to necrosis area ${ }^{(2)}$

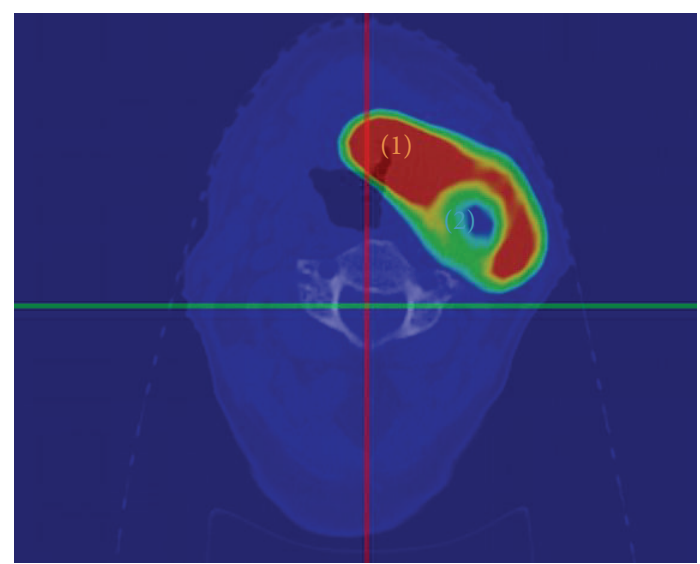

(b)

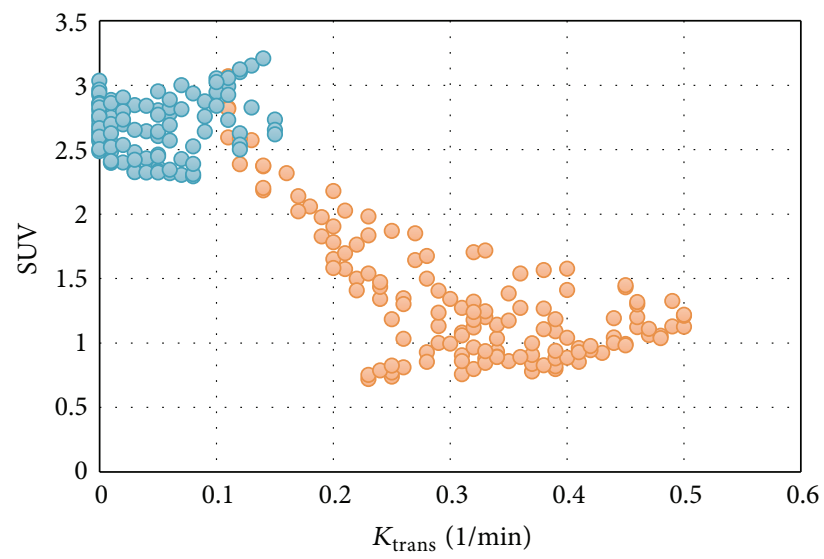

Tumor away to necrosis area ${ }^{(1)}$

- Near to necrosis area ${ }^{(2)}$

(d)

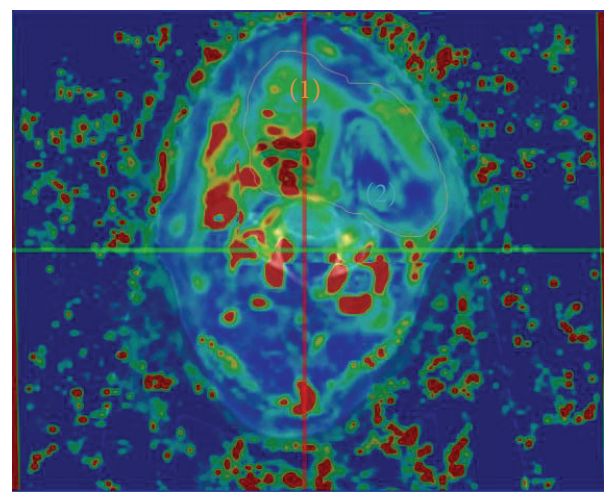

(e)

FIGURE 5: In this figure SUV versus $K_{\text {trans }}$ and ADC versus $K_{\text {trans }}$ are represented. (a) $K_{\text {trans }}$ map overlaid on the simulation CT. (b) PET/CT. (c) In the hypoxic area (near necrotic area), high SUV values are obtained independently for all low $K_{\text {trans }}$ values, because of the addition of the Warburg effect and the Pasteur effect. In the heterogeneously vascularized area, SUV values are decreasing with $K_{\text {trans }}$, as expected, because the Pasteur effect is reducing in this area as $K_{\text {trans }}$ increases. (d) No clear relationship can be found between ADC and $K_{\text {trans }}$. (e) ADC map overlaid simulation CT. 


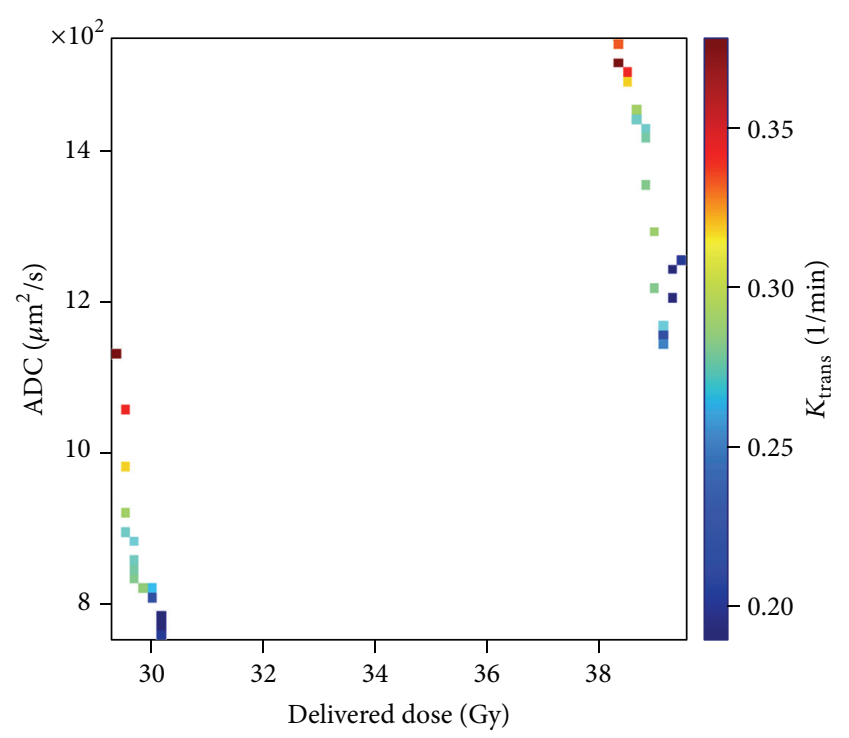

FIGURE 6: ADC values for a heterogeneously vascularized tumour volume are represented versus delivered dose (fractions 13th and 17 th), and the colour represents the $k_{\text {trans }}$ value. In this graph, it can be observed that heterogeneously vascularized voxels show a greater increment in $\mathrm{ADC}$ values.

ADC values can be a good marker of the tumour response $[11,12]$. Further, the combination of biological information obtained from different modalities can improve the characterization of tumour behaviour. From our point of view, at least two different sets of data must be considered: one for tumour response and another one for hypoxia measurement. If geometrical distortion is not considered or can be corrected [60], ADC maps can be a suitable choice for tumour response.

The polarographic electrode has been considered by some authors as the gold standard for measuring tumour hypoxia in vivo [61], although theoretical simulations have shown that it gives only a qualitative characterisation. Considering only radiopharmaceuticals and PET/CT, the most common are FMISO [24, 26, 32, 33], dynamic FDG [28], and $\mathrm{Cu}-$ ATSM [47]. When considering MRI, typically BOLD [17] and DCE-MRI $[19,20,22,62]$ are the most widely used methods; however we have not found any study using them for modifying the treatment (as with FMISO [26, 33, 63]). Vascularity measurements from DCE-MRI data can provide a surrogate marker of tumour hypoxia, as was shown by Newbold et al. [20] and Donaldson et al. [21] in head and neck cancer. These measurements could potentially guide treatment [22] and are easy to obtain; however more studies are needed in order to apply to clinical practice, as input data either for dose painting or for delimiting hypoxic volumes.

18F-FDG shows different aspects of the tumour behaviour, mainly associated with tumour cell density, malignancy, and oxygenation, and the quotient between ADC and SUV has been proposed as a measurement of malignancy in breast tumours [64] and in invasive ductal cancer [65]. These last papers found correlation between maximum SUV and bad prognoses that could be explained because high SUV can be associated with hypoxic areas as we have observed.
Using biomechanical models [66] that consider both the dynamics of the tumour and variation of tumour density (including diffusion) and oxygenation along the treatment, instead of static models, can be quite useful for increasing the predictability of the models.

ADC maps appear to be a good option for evaluating tumour response; however their disadvantage is image distortion. Unfortunately, this cannot be corrected using standard deformable registration algorithm, but reversed gradients method looks like a very promising algorithm to solve this problem [60]. It is possible that extracellular volume calculated from DCE-MRI can be used as an equivalent of ADC values in well-vascularized areas.

\section{Conclusions}

Multimodality imaging offers much more information about tumour behaviour than the individual datasets on their own. The relationship between different types of images must be studied in detail in order to establish a minimum set of data required to personalize the radiotherapy treatment and to optimize the treatment for each patient. This could comprise not only a gradient of dose along the treatment, but also different fractionation for each voxel.

Multicentre studies can be useful for recruitment of a large number of patients and increase the statistical power of the results, if imaging standards and protocol compliance are followed [67].

Voxel by voxel analysis seems possible if we consider small volumes and undistorted regions from ADC maps or corrected data.

\section{Conflict of Interests}

The authors declare that there is no conflict of interests regarding the publication of this paper.

\section{Acknowledgments}

The authors offer their sincerest thanks to patients who volunteered to participate in this study. The authors also would like to acknowledge Neil Burnet and Michael Simmons for their helpful comments about this text and the editorial committee for inviting them to collaborate in this special issue. The authors must be grateful for the exchange program of the Spanish Society of Medical Physics (SEFM) that funded the first author's visit to Princess Margaret Cancer Centre. The authors thank the National Health Institute of Spain for supporting this work with ISCIII Grant PI11/02035 and the Galician Government through Project CN 2012/260 "Consolidation Research Units: AtlantTIC."

\section{References}

[1] C. Grau, M. Hoyer, M. Alber, J. Overgaard, J. C. Lindegaard, and L. P. Muren, "Biology-guided adaptive radiotherapy (BiGART)-more than a vision?" Acta Oncologica, vol. 52, no. 7, pp. 1243-1247, 2013. 
[2] K. Smit, B. van Asselen, J. G. M. Kok, A. H. L. Aalbers, J. J. W. Lagendijk, and B. W. Raaymakers, "Towards reference dosimetry for the MR-linac: magnetic field correction of the ionization chamber reading," Physics in Medicine and Biology, vol. 58, no. 17, pp. 5945-5957, 2013.

[3] D. Saenz, B. Paliwal, and J. Bayouth, "A dose homogeneity and conformity evaluation between ViewRay and pinnacle-based linear accelerator IMRT treatment plans," Journal of Medical Physics, vol. 39, no. 2, pp. 64-70, 2014.

[4] J. Winter, M. Westmore, M. Dahan et al., "Patient alignment in MRI guided radiation therapy," US Patent 20130235969 A1, 2013.

[5] B. W. Raaymakers, J. J. W. Lagendijk, J. Overweg et al., "Integrating a $1.5 \mathrm{~T}$ MRI scanner with a $6 \mathrm{MV}$ accelerator: proof of concept," Physics in Medicine and Biology, vol. 54, no. 12, pp. N229-N237, 2009.

[6] V. Grégoire, K. Haustermans, X. Geets, S. Roels, and M. Lonneux, "PET-based treatment planning in radiotherapy: a new standard?" Journal of Nuclear Medicine, vol. 48, supplement 1, pp. 68S-77S, 2007.

[7] M. Lonneux, M. Hamoir, H. Reychler et al., "Positron emission tomography with $\left[{ }^{18} \mathrm{~F}\right]$ fluorodeoxyglucose improves staging and patient management in patients with head and neck squamous cell carcinoma: a multicenter prospective study," Journal of Clinical Oncology, vol. 28, no. 7, pp. 1190-1195, 2010.

[8] M. MacManus, U. Nestle, K. E. Rosenzweig et al., "Use of PET and PET/CT for radiation therapy planning: IAEA expert report 2006-2007," Radiotherapy and Oncology, vol. 91, no. 1, pp. 85-94, 2009.

[9] D. A. Hamstra, T. L. Chenevert, B. A. Moffat et al., "Evaluation of the functional diffusion map as an early biomarker of timeto-progression and overall survival in high-grade glioma," Proceedings of the National Academy of Sciences of the United States of America, vol. 102, no. 46, pp. 16759-16764, 2005.

[10] C. J. Galbán, S. K. Mukherji, T. L. Chenevert et al., "A feasibility study of parametric response map analysis of diffusionweighted magnetic resonance imaging scans of head and neck cancer patients for providing early detection of therapeutic efficacy," Translational Oncology, vol. 2, no. 3, pp. 184-190, 2009.

[11] S. Kim, L. Loevner, H. Quon et al., "Diffusion-weighted magnetic resonance imaging for predicting and detecting early response to chemoradiation therapy of squamous cell carcinomas of the head and neck," Clinical Cancer Research, vol. 15, no. 3, pp. 986-994, 2009.

[12] N. C. Atuegwu, J. C. Gore, and T. E. Yankeelov, "The integration of quantitative multi-modality imaging data into mathematical models of tumors," Physics in Medicine and Biology, vol. 55, no. 9, pp. 2429-2449, 2010.

[13] L. A. P. Romasanta, M. J. G. Velloso, and A. L. Medina, "Functional imaging in radiation therapy planning for head and neck cancer," Reports of Practical Oncology and Radiotherapy, vol. 18, no. 6, pp. 376-382, 2013.

[14] J. M. Arbeit, J. M. Brown, K. S. C. Chao et al., "Hypoxia: importance in tumor biology, noninvasive measurement by imaging, and value of its measurement in the management of cancer therapy," International Journal of Radiation Biology, vol. 82, no. 10, pp. 699-757, 2006.

[15] A. R. Padhani, K. A. Krohn, J. S. Lewis, and M. Alber, "Imaging oxygenation of human tumours," European Radiology, vol. 17, no. 4, pp. 861-872, 2007.
[16] Y. Cao, C. I. Tsien, V. Nagesh et al., "Clinical investigation survival prediction in high-grade gliomas by MRI perfusion before and during early stage of RT,' International Journal of Radiation Oncology Biology Physics, vol. 64, no. 3, pp. 876-885, 2006.

[17] J. F. Dunn, J. A. O'Hara, Y. Zaim-Wadghiri et al., "Changes in oxygenation of intracranial tumors with carbogen: a BOLD MRI and EPR oximetry study," Journal of Magnetic Resonance Imaging, vol. 16, no. 5, pp. 511-521, 2002.

[18] M. A. Zahra, K. G. Hollingsworth, E. Sala, D. J. Lomas, and L. T. Tan, "Dynamic contrast-enhanced MRI as a predictor of tumour response to radiotherapy," The Lancet Oncology, vol. 8, no. 1, pp. 63-74, 2007.

[19] J. T. Elliott, E. A. Wright, K. M. Tichauer et al., "Arterial input function of an optical tracer for dynamic contrast enhanced imaging can be determined from pulse oximetry oxygen saturation measurements," Physics in Medicine and Biology, vol. 57, no. 24, pp. 8285-8295, 2012.

[20] K. Newbold, I. Castellano, E. Charles-Edwards et al., "An exploratory study into the rol of dynamic contrast-enhanced magnetic resonance imaging or perfusion computed tomography for detection of intratumoral hypoxia in head and neck cancer," International Journal of Radiation Oncology Biology Physics, vol. 74, no. 1, pp. 29-37, 2009.

[21] S. B. Donaldson, G. Betts, S. C. Bonington et al., "Perfusion estimated with rapid dynamic contrast-enhanced magnetic resonance imaging correlates inversely with vascular endothelial growth factor expression and pimonidazole staining in head-and-neck cancer: a pilot study," International Journal of Radiation Oncology Biology Physics, vol. 81, no. 4, pp. 1176-1183, 2011.

[22] J. M. Berstein, J. J. Homer, and C. M. West, "Dynamic contrastenhanced magnetic resonance imaging biomarkers in head and neck cancer: potential to guide treatment? A systematic review," Oral Oncology, vol. 50, no. 10, pp. 963-970, 2014.

[23] M. Busk, M. R. Horsman, S. Jakobsen et al., "Can hypoxiaPET map hypoxic cell density heterogeneity accurately in an animal tumor model at a clinically obtainable image contrast?" Radiotherapy and Oncology, vol. 92, no. 3, pp. 429-436, 2009.

[24] N. Y. Lee, J. G. Mechalakos, S. Nehmeh et al., "Fluorine-18labeled fluoromisonidazole positron emission and computed tomography-guided intensity-modulated radiotherapy for head and neck cancer: a feasibility study," International Journal of Radiation Oncology, Biology, Physics, vol. 70, no. 1, pp. 2-13, 2008.

[25] S. A. Nehmeh, N. Y. Lee, H. Schröder et al., "Reproducibility of intratumor distribution of (18)F-fluoromisonidazole in head and neck cancer," International Journal of Radiation Oncology Biology Physics, vol. 70, no. 1, pp. 235-242, 2008.

[26] D. Thorwarth, S.-M. Eschmann, F. Paulsen, and M. Alber, "Hypoxia dose painting by numbers: a planning study," International Journal of Radiation Oncology Biology Physics, vol. 68, no. 1, pp. 291-300, 2007.

[27] S.-M. Eschmann, F. Paulsen, M. Reimold et al., "Prognostic impact of hypoxia imaging with ${ }^{18} \mathrm{~F}$-misonidazole PET in nonsmall cell lung cancer and head and neck cancer before radiotherapy," Journal of Nuclear Medicine, vol. 46, no. 2, pp. 253-260, 2005.

[28] K. Røe, T. B. Aleksandersen, A. Kristian et al., "Preclinical dynamic 18F-FDG PET tumor characterization and radiotherapy response assessment by kinetic compartment analysis," Acta Oncologica, vol. 49, no. 7, pp. 914-921, 2010. 
[29] V. A. Semenenko, B. Reitz, E. Day, X. S. Qi, M. Miften, and X. A. Li, "Evaluation of a commercial biologically based IMRT treatment planning system," Medical Physics, vol. 35, no. 12, pp. 5851-5860, 2008.

[30] P. Stavrev, D. Hristov, B. Warkentin, E. Sham, N. Stavreva, and B. G. Fallone, "Inverse treatment planning by physically constrained minimization of a biological objective function," Medical Physics, vol. 30, no. 11, pp. 2948-2958, 2003.

[31] J. Heukelom, O. Hamming, H. Bartelink et al., "Adaptive and innovative Radiation Treatment FOR improving Cancer treatment outcomE (ARTFORCE); a randomized controlled phase II trial for individualized treatment of head and neck cancer," BMC Cancer, vol. 13, article 84, 2013.

[32] D. Thorwarth and M. Alber, "Implementation of hypoxia imaging into treatment planning and delivery," Radiotherapy and Oncology, vol. 97, no. 2, pp. 172-175, 2010.

[33] I. Toma-Dasu, J. Uhrdin, L. Antonovic et al., "Dose prescription and treatment planning based on FMISO-PET hypoxia," Acta Oncologica, vol. 51, no. 2, pp. 222-230, 2012.

[34] A. López Medina, D. Aramburu, M. Mera et al., "ARTFIBio project: quantifying tumour response voxel by voxel," Radiotherapy \& Oncology, vol. 106, p. S329, 2013.

[35] A. L. Medina, D. Aramburu, M. Mera et al., "Tumour response: a multiparametric function," Radiotherapy \& Oncology, vol. 111, supplement 1, pp. 149-150, 2014.

[36] I. Landesa-Vázquez, J. Alba-Castro, M. Mera-Iglesias, D. Aramburu-Núñez, A. López-Medina, and V. Muñóz-Garzón, “ARTFIBio: a cross-platform image registration tool for tumor response quantification in head and neck cancer," in Proceedings of the IEEE-EMBS International Conference on Biomedical and Health Informatics (BHI '14), pp. 149-152, Valencia, Spain, June 2014.

[37] O. Warburg, "On the origin of cancer cells," Science, vol. 123, no. 3191, pp. 309-314, 1956.

[38] T. Roose, S. J. Chapman, and P. K. Maini, "Mathematical models of avascular tumor growth," SIAM Review, vol. 49, no. 2, pp. 179208, 2007.

[39] M. Busk, M. R. Horsman, P. E. G. Kristjansen, A. J. van der Kogel, J. Bussink, and J. Overgaard, "Aerobic glycolysis in cancers: implications for the usability of oxygen-responsive genes and fluorodeoxyglucose-PET as markers of tissue hypoxia," International Journal of Cancer, vol. 122, no. 12, pp. 2726-2734, 2008.

[40] R. A. Gatenby, E. T. Gawlinski, A. F. Gmitro, B. Kaylor, and R. J. Gillies, "Acid-mediated tumor invasion: a multidisciplinary study," Cancer Research, vol. 66, no. 10, pp. 5216-5223, 2006.

[41] E. O. Stejskal and J. E. Tanner, "Spin diffusion measurements: spin echoes in the presence of a time-dependent field gradient," The Journal of Chemical Physics, vol. 42, no. 1, pp. 288-292, 1965.

[42] D.-M. Koh and D. J. Collins, "Diffusion-weighted MRI in the body: applications and challenges in oncology," American Journal of Roentgenology, vol. 188, no. 6, pp. 1622-1635, 2007.

[43] A. C. Morani, K. M. Elsayes, P. S. Liu et al., "Abdominal applications of diffusion-weighted magnetic resonance imaging: where do we stand?" World Journal of Radiology, vol. 5, no. 3, pp. 6880, 2013.

[44] J. F. Kallehauge, C. Nomden, and A. C. S. de Castro, “Temporal changes in DCE-MRI parameters during treatment of locally advanced cervical cancer," Radiotherapy \& Oncology, vol. 103, p. S78, 2012.
[45] D. S. Yoo, J. P. Kirkpatrick, O. Craciunescu et al., "Prospective trial of synchronous bevacizumab, erlotinib, and concurrent chemoradiation in locally advanced head and neck cancer," Clinical Cancer Research, vol. 18, no. 5, pp. 1404-1414, 2012.

[46] D. Zheng, Y. Chen, X. Liu et al., "Early response to chemoradiotherapy for nasopharyngeal carcinoma treatment: value of dynamic contrast-enhanced 3.0 T MRI," Journal of Magnetic Resonance Imaging, 2014.

[47] B. Titz and R. Jeraj, "An imaging-based tumour growth and treatment response model: investigating the effect of tumour oxygenation on radiation therapy response," Physics in Medicine and Biology, vol. 53, no. 17, pp. 4471-4488, 2008.

[48] World Medical Organization, "Declaration of Helsinki," British Medical Journal, vol. 313, no. 7070, pp. 1448-1449, 1996.

[49] http://www.thedcetool.com/scientific.

[50] P. S. Tofts, "Modeling tracer kinetics in dynamic Gd-DTPA MR imaging," Journal of Magnetic Resonance Imaging, vol. 7, no. 1, pp. 91-101, 1997.

[51] C. Coolens, B. Driscoll, C. Chung et al., "Automated voxel-based analysis of volumetric DCE CT data improves the measurement of serial changes in tumor vascular biomarkers," International Journal of Radiation Oncology, Biology, Physics. In press.

[52] W. Foltz, B. Driscoll, S. J. Lee et al., "Comparison of arterial input functions by magnitude and phase signal measurement in dynamic contrast enhancement MRI using a dynamic flow phantom," Medical Physics. In press.

[53] IAEA, Quantitative Nuclear Medicine Imaging: Concepts, Requirements and Methods, IAEA Library, 2014.

[54] En línea, http://www.itk.org/.

[55] T. W. Secomb, R. Hsu, M. W. Dewhirst, B. Klitzman, and J. F. Gross, "Analysis of oxygen transport to tumor tissue by microvascular networks," International Journal of Radiation Oncology, Biology, Physics, vol. 25, no. 3, pp. 481-489, 1993.

[56] C. J. Kelly and M. Brady, "A model to simulate tumour oxygenation and dynamic [18F]-Fmiso PET data," Physics in Medicine and Biology, vol. 51, no. 22, pp. 5859-5873, 2006.

[57] S. S. Foo, D. F. Abbott, N. Lawrentschuk, and A. M. Scott, "Functional imaging of intratumoral hypoxia," Molecular Imaging and Biology, vol. 6, no. 5, pp. 291-305, 2004.

[58] R. A. Cooper, B. M. Carrington, J. A. Loncaster et al., “Tumour oxygenation levels correlate with dynamic contrast-enhanced magnetic resonance imaging parameters in carcinoma of the cervix," Radiotherapy and Oncology, vol. 57, no. 1, pp. 53-59, 2000.

[59] H. Lyng, A. O. Vorren, K. Sundfør et al., "Assessment of tumor oxygenation in human cervical carcinoma by use of dynamic Gd-DTPA-enhanced MR imaging," Journal of Magnetic Resonance Imaging, vol. 14, no. 6, pp. 750-756, 2001.

[60] P. S. Morgan, R. W. Bowtell, D. J. O. McIntyre, and B. S. Worthington, "Correction of spatial distortion in EPI due to inhomogeneous static magnetic fields using the reversed gradient method," Journal of Magnetic Resonance Imaging, vol. 19, no. 4, pp. 499-507, 2004.

[61] J. G. Eriksen and M. R. Horsman, "Tumour hypoxia-a characteristic feature with a complex molecular background," Radiotherapy and Oncology, vol. 81, no. 2, pp. 119-121, 2006.

[62] T. E. Yankeelov, M. Lepage, A. Chakravarthy et al., "Integration of quantitative DCE-MRI and ADC mapping to monitor treatment response in human breast cancer: initial results," Magnetic Resonance Imaging, vol. 25, no. 1, pp. 1-13, 2007. 
[63] P. Dirix, V. Vandecaveye, F. De Keyzer, S. Stroobants, R. Hermans, and S. Nuyts, "Dose painting in radiotherapy for head and neck squamous cell carcinoma: value of repeated functional imaging with 18F-FDG PET, 18F-fluoromisonidazole PET, diffusion-weighted MRI, and dynamic contrast-enhanced MRI," Journal of Nuclear Medicine, vol. 50, no. 7, pp. 1020-1027, 2009.

[64] S. Baba, T. Isoda, Y. Maruoka et al., "Diagnostic and prognostic value of pretreatment SUV in ${ }^{18} \mathrm{~F}-\mathrm{FDG} / \mathrm{PET}$ in breast cancer: comparison with apparent diffusion coefficient from diffusionweighted MR imaging," Journal of Nuclear Medicine, vol. 55, no. 5, pp. 736-742, 2014.

[65] B. B. Choi, S. H. Kim, B. J. Kang et al., "Diffusion-weighted imaging and FDG PET/CT: predicting the prognoses with apparent diffusion coefficient values and maximum standardized uptake values in patients with invasive ductal carcinoma," World Journal of Surgical Oncology, vol. 10, article 126, 2012.

[66] J. A. Weis, M. I. Miga, L. R. Arlinghaus et al., "A mechanically coupled reaction-diffusion model for predicting the response of breast tumors to neoadjuvant chemotherapy," Physics in Medicine and Biology, vol. 58, no. 17, pp. 5851-5866, 2013.

[67] B. Driscoll, H. Keller, D. Jaffray, and C. Coolens, "Development of a dynamic quality assurance testing protocol for multisite clinical trial DCE-CT accreditation," Medical Physics, vol. 40, no. 8, Article ID 081906, 2013. 


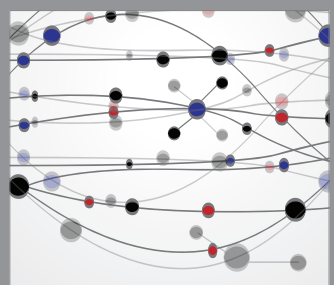

The Scientific World Journal
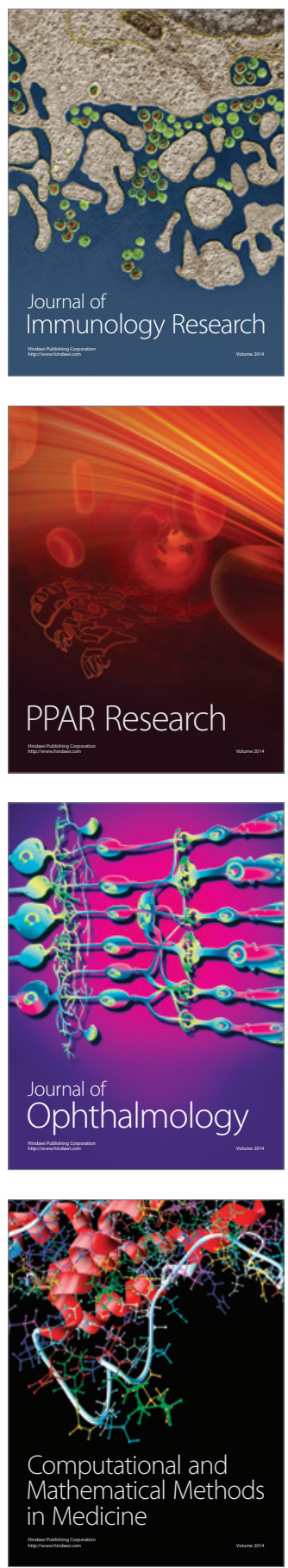

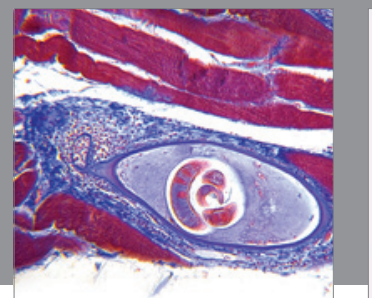

Gastroenterology

Research and Practice
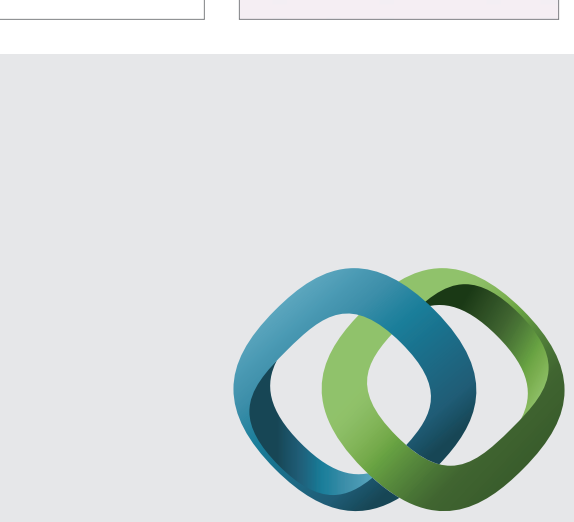

\section{Hindawi}

Submit your manuscripts at

http://www.hindawi.com
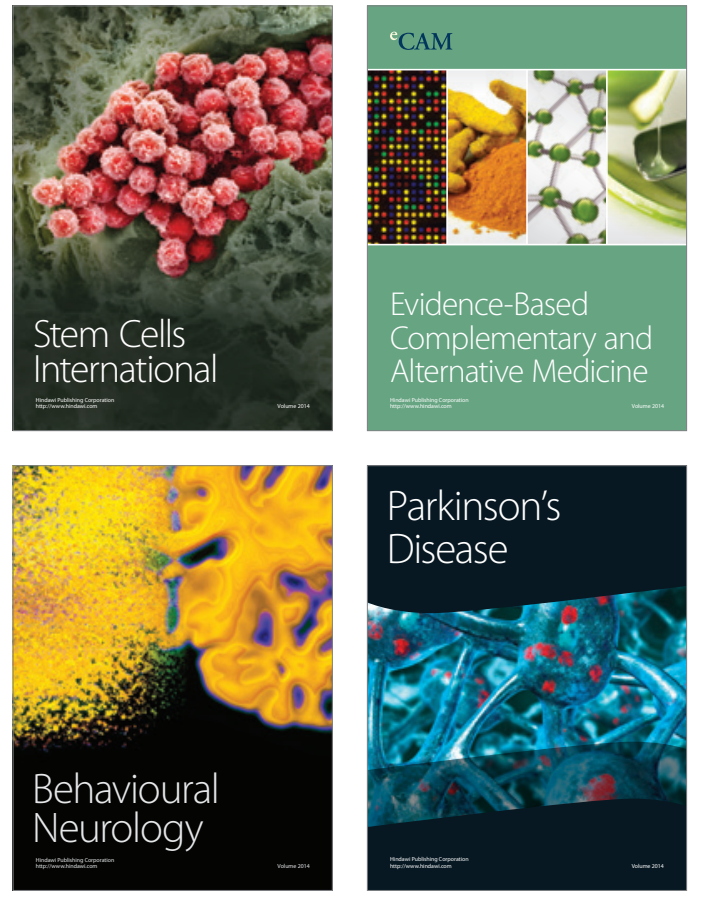
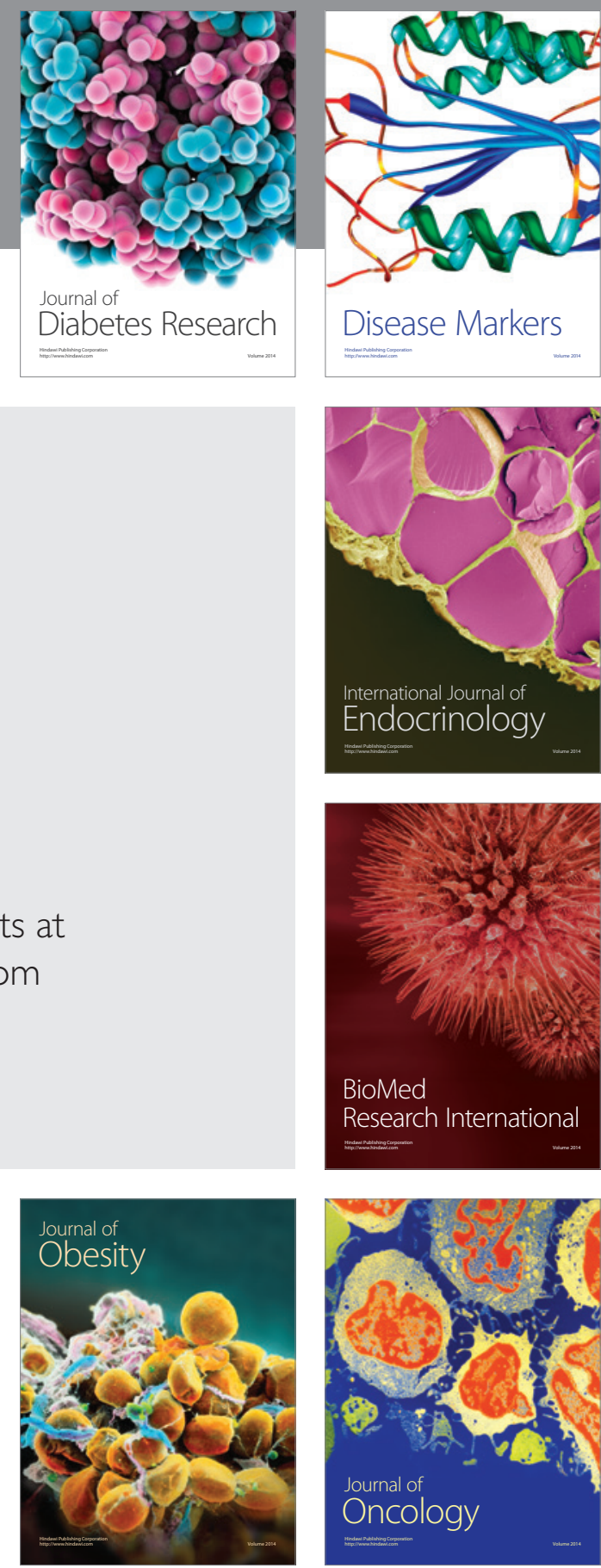

Disease Markers
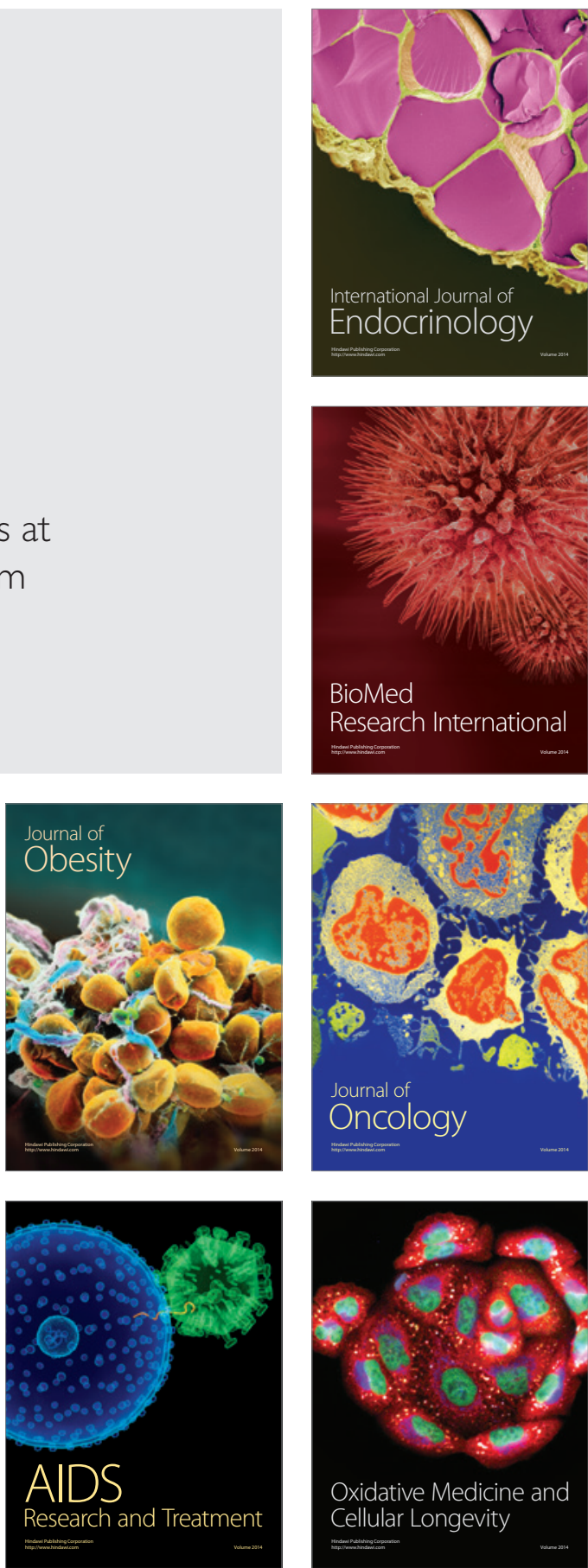